\title{
Early Disruption of Synaptic Function, Impairment of Plasticity, and Decreased of Cortical Circuit Connectivity in an Alzheimer's Mouse Model of Amyloid Deposition
}

\section{Chang Chen}

Nanjing University Medical School Affiliated Nanjing Drum Tower Hospital

Jing Wei

The University of Arizona College of Medicine Phoenix

\section{Xiaokuang $\mathrm{Ma}$}

The University of Arizona College of Medicine Phoenix

\section{Le Zhang}

The University of Arizona College of Medicine Phoenix

\section{Antoine Nehme}

The University of Arizona College of Medicine Phoenix

\section{Yuehua Cui}

The University of Arizona College of Medicine Phoenix

\section{Deveroux Ferguson}

The University of Arizona College of Medicine Phoenix

\section{Shenfeng Qiu}

The University of Arizona College of Medicine Phoenix

\section{Feng Bai ( $\nabla$ baifeng@njglyy.com )}

Nanjing University Medical School Affiliated Nanjing Drum Tower Hospital https://orcid.org/00000001-7938-4729

\section{Research}

Keywords: Alzheimer's disease, mouse model, synaptic plasticity, long-term potentiation, cortical circuit, electrophysiology, learning and memory

Posted Date: November 4th, 2021

DOl: https://doi.org/10.21203/rs.3.rs-948437/v1

License: (c) (1) This work is licensed under a Creative Commons Attribution 4.0 International License. 



\section{Early disruption of synaptic function, impairment of plasticity, and decreased of cortical circuit connectivity in an Alzheimer's mouse model of amyloid deposition}

Chang Chen ${ }^{1,2}$, Jing $\mathrm{Wei}^{2}$, Xiaokuang $\mathrm{Ma}^{2}$, Le Zhang ${ }^{2}$, Antoine Nehme ${ }^{2}$, Yuehua Cui ${ }^{2}$, Deveroux Ferguson $^{2}$, Shenfeng $\mathrm{Qiu}^{2 *}$, Feng Bai ${ }^{*}$

1. Department of Neurology, Affiliated Drum Tower Hospital, Medical School of Nanjing University, Nanjing, Jiangsu, 210008, China.

2. Basic Medical Sciences, University of Arizona College of Medicine-Phoenix, Phoenix, Arizona, 85004, USA.

* Corresponding author:

Feng Bai, baifeng515@126.com

Shenfeng Qiu, sqiu@arizona.edu 


\begin{abstract}
Mutations in genes encoding amyloid precursor proteins and presenilins lead to increased $\beta$-amyloid (A $\beta$ ) production and cause familial Alzheimer's disease (AD), a neurodegenerative disorder often associates with aging and features synapse loss and impaired synaptic plasticity. $A \beta$ deposition is a pathological hallmark of $A D$. It is currently unknown whether and how $A D$ risk alleles affects development of brain circuit function, and whether subtle synaptic pathology occurs prior to overt A $\beta$ deposition. Transgenic mutated APP/PS1 over-expression mice lines are key tools to study molecular mechanisms of AD pathogenesis. Among these mice lines, the SXFAD mice rapidly recapitulate key features of AD pathology, and have proven utility in studying amyloid plaque formation and $A \beta$-induced neurodegeneration. We reason that transgenic mutant APP/PS1 over-expression may lead to neurodevelopmental defects in early cortical neurons as a result of continuous APP/A $\beta$ expression from early life. We first explored agedependent plasticity changes in prefrontal cortical circuits in the 5XFAD mice, and found that at an early age (6-8 wks old) that does not produce overt impairment of hippocampus LTP, layer (L) 5 neuron circuit plasticity, measured by long term potentiation (LTP), is impaired. In addition, L5 neurons, which are reportedly vulnerable cortical neuron populations, show reduced mEPSC amplitude and frequency in early postweaning ages, indicating impaired synaptic transmission as a result of transgenic APP/A $\beta$ overloading during early postnatal development. These functional changes were corroborated by the morphological findings of smaller, sparser dendritic spines on L5 pyramidal neurons, indicative of impaired prefrontal circuit function. Lastly, laser scanning photostimulation (LSPS) combined with glutamate uncaging revealed that $\mathrm{L} 2 / 3>\mathrm{L} 5$ cortical connectivity was decreased at this early age. Our results suggest $5 \mathrm{XFAD}$ transgenic mutant APP/PS1 over expression causes developmental defects of cortical circuits, which could contribute to the age-dependent synaptic pathology and neurodegeneration later in life.
\end{abstract}

KEYWORDS: Alzheimer's disease, mouse model, synaptic plasticity, long-term potentiation, cortical circuit, electrophysiology, learning and memory 


\section{INTRODUCTION}

Alzheimer's disease (AD) represents the most frequent form of dementia. A hallmark pathology of the $A D$ brain is the degenerating cortical neurons overloaded with neurofibrillary tangles and amyloid plagues ${ }^{1}$. At cellular and functional levels, early AD brain is characterized by impaired synaptic function and synapse loss, manifested as disrupted synaptic plasticity, learning, memory, and cognition ${ }^{2}$. Transgenic mice that overexpress the mutated human amyloid precursor protein (APP), presenilin (PS) and tau genes reproduce many aspects of AD pathology such as $\beta$-amyloid plaques $(A \beta)$, neurofibrillary tangles, reactive gliosis, synaptic and neuronal loss, which are consistent with behavioral changes such as progressive age-dependent memory impairments ${ }^{3}$. As such, these mice line are useful to inform molecular and cellular changes associated with $A D$.

The 5XFAD mouse line, which co-overexpresses human APP and presenilin 1 (PS1) harboring five familial AD (FAD) mutations manifest very early-onset and aggressive amyloid deposition ${ }^{4,5}$; These mice start to develop amyloid plaques as early as 2 months of age, at which they show dramatically accelerated intraneuronal $A \beta 42$ production. Pathologically, $A \beta$ deposition emerges in the hippocampus subiculum and in layer 5 (L5) principal neurons of the neocortex, and rapidly increases in affected brain regions ${ }^{4}$. Synaptic failures reportedly taken place prior to overt plaque formation; for example, it has been shown that the hippocampal basal synaptic transmission and theta burst stimulus (TBS)-induced LTP is impaired at 6 months, but not prior to 4 months ${ }^{6}$. In addition, synaptic failure can manifest at multiple levels, ranging from functional alterations to structural disturbances ${ }^{7}$. Synaptic failure of $L 5$ neurons in $5 x F A D$ mice reportedly long precede their physical loss, which happens by 12 months of age ${ }^{7}$.

Although $A D$ is a disease associated with aging, the mutant forms APP/PS1 is expected to be expressed throughout life span. As such, how transgenic over-expression of mutant forms APP/PS1 affects the developing neural circuit function may be an interesting question. The 5XFAD mouse line, which utilizes Thy1 promoter (Bradley, Ramirez, \& Hagood, 2009) to drive transgenic APP/PS1 expression, offers a unique opportunity to study neurodevelopmental 
effects of APP/PS1 over-loading in the affected neuron populations. Despite a large literature exploring mechanisms of neurodegeneration in 5XFAD mice ${ }^{8-11}$, in this study we focused on early developmental synaptic deficits resulting from mutated forms of APP/PS1. Because Thy1promotor driven expression of mutated forms of APP/PS1 are expected to predominantly affect layer 5 neurons ${ }^{7,12,13}$, we reason that mutated transgenic APP/PS1 and the processed intracellular $A \beta$ may appear at an much early time point, thus impairing normal development of synapse function and connectivity at very early age. In this study, we provide electrophysiology evidence of impaired synaptic activity, plasticity, and decreased of cortical circuit connectivity in the L5 prefrontal cortex neurons at an early postweaning age (P25-35). Our data suggest that transgenic mutant APP/PS1 over-loading in the 5XFAD mouse model leads to impaired early cortical circuit development, an underappreciated neuropathology in this prevalent AD mouse model.

\section{MATERIALS AND METHODS}

\section{Animals}

The 5XFAD mice used in this study were obtained from Jackson laboratory (catalog \#34840-JAX), and maintained on the B6SJLF1 background. This mouse line overexpresses both human amyloid precursor protein (APP) gene with K670N/M671L, V717I, and I716V mutations and the human PS1 M146L and L286V mutations under the Thy1 promoter ${ }^{4}$. Mice were genotyped according to JAX protocol using three primers ('mutant', AAG CTA GCT GCA GTA ACG CCA TTT ; 'wild type', ACC TGC ATG TGA ACC CAG TAT TCT ATC; and 'common', CTA CAG CCC CTC TCC AAG GTT TAT AG). Mice were weaned at P21 and used for experiments at ages postnatal day (PD) 11- 6 months. All experimental procedures conforms to $\mathrm{NIH}$ guidelines and were approved by the Institutional Animal Care and Use Committee of the University of Arizona and the Animal Care and Use Committee of the Model Animal Research Center, Nanjing University.

\section{Field potential recording and synaptic plasticity}

Mice of desired genotypes were anesthetized using 3-5\% isoflurane. To improve brain slice viability, intra-cardiac perfusion of ice-cold choline solution (in mM: 110 choline chloride, $25 \mathrm{NaHCO}_{3}, 2.5 \mathrm{KCl}, 1.25 \mathrm{NaH}_{2} \mathrm{PO}_{4}, 0.5 \mathrm{CaCl}_{2}, 7$ $\mathrm{MgSO}_{4}, 25 \mathrm{D}$-glucose, 11.6 sodium ascorbate, and 3.1 sodium pyruvate, saturated with $95 \% \mathrm{O}_{2} / 5 \% \mathrm{CO}_{2}$ ) was performed before mice were decapitated and brains quickly dissected out. To prepare prefrontal slices (350 $\mu \mathrm{m}$ thick), we used parasagittal sections, which allows better preservation of intracortical synaptic connectivity ${ }^{14}$. To prepare the hippocampus slices, horizontal sections (300 $\mu$ m thick) at the middle septotemporal levels were made. Slices were cut 
in ice-cold choline solution using a vibratome (VT-1200S, Leica). Both PFC and hippocampus slices were kept in artificial cerebrospinal fluid (ACSF, contains in mM: $126 \mathrm{NaCl}, 2.5 \mathrm{KCl}, 26 \mathrm{NaHCO}_{3}, 2 \mathrm{CaCl}_{2}, 2 \mathrm{MgCl}_{2}, 1.25 \mathrm{NaH}_{2} \mathrm{PO}_{4}$, and $10 \mathrm{~d}$-glucose; saturated with $95 \% \mathrm{O}_{2} / 5 \% \mathrm{CO}_{2}$ ) for $30 \mathrm{~min}$ at $35^{\circ} \mathrm{C}$, and then maintained at $24^{\circ} \mathrm{C}$ RT until recording.

The brain slices were transferred to an interface chamber (AutoMate Scientific) to facilitate long-term slice viability, and superfused with ACSF saturated with $95 \% \mathrm{O}_{2} / 5 \% \mathrm{CO}_{2}$. Field excitatory postsynaptic potentials (fEPSPs) were recorded using a glass patch electrode in L5 pyramidal neuron layer (mPFC recording, in response to L2/3 stimulation) or in the CA1 stratum radiatum layer (hippocampus recording, in response to Schaffer collateral stimulation). The patch electrode had an electrical resistance of $1-2 \mathrm{M} \Omega$ at $1 \mathrm{kHz}$ when filled with ACSF. Electric stimuli were delivered by a bipolar tungsten electrode (FHC, Bowdoin, ME) that was placed 200 $\mu \mathrm{m}$ away from the recording site, using biphasic stimuli (10-250 $\mu \mathrm{A}, 100 \mu \mathrm{s}$ duration, $0.05 \mathrm{~Hz}$ for baseline recording). Stimulus was generated using a Digidata 1440A (Molecular Devices, San Jose, CA) device, and delivered through an optic isolator (Iso-flex, A.M.P.I). fEPSP signals were amplified using a differential amplifier (model 1800, A-M Systems, Carlsborg, WA), low-pass filtered at 2 $\mathrm{kHz}$ and digitized at $10 \mathrm{kHz}$.

For fEPSP recordings from both mPFC and HPC, a stimulus-response (input-output) curve was obtained by measuring fEPSP slope (first 1-ms response after fiber volley) as a function of the fiber volley amplitude, which is used to quantify basal synaptic transmission strength. We then chose a stimulus intensity produces a $\sim 40-50 \%$ maximum fEPSP amplitude throughout the experiments. Following a 10min stable baseline response of stimulus-evoked fEPSPs, we tested paired-pulse responses at inter-pulse intervals ranging from $20-200 \mathrm{~ms}$ in order to probe potential changes in presynaptic transmission. An LTP induction stimulation protocol was then applied. Because stimulation pattern is critical for LTP/LTD induction and maintenance ${ }^{15-19}$, we adopted a tetanus stimulation protocol that consisted of two, 1-sec trains of stimulation at $100 \mathrm{~Hz}{ }^{20}$ for both mPFC and CA1 LTP induction. Following LTP induction, fEPSP responses were recorded for an additional 1 hour.

\section{Whole cell recording in brain slices}

Whole cell recordings were conducted in L 5 pyramidal neurons in parasagittal mPFC slices, which are prepared similarly to slices used for fEPSP recordings, except they are perfused with 95\%O2/5\% CO2-saturated ACSF in a submerged chamber during recording. Slices were visualized under a $4 \mathrm{X}$ objective (Olympus UPlanApo, NA=0.16) to locate the cytoarchitectural landmarks of layer 5 . To minimize neurite cutoffs, only L5 pyramidal neurons with soma at least $50 \mu \mathrm{m}$ below the slice surface were selected for whole cell recordings. Neuronal soma were identified and targeted using a $60 \mathrm{X}$ objective (NA = 0.9) under IR illumination (Olympus BX-51 WI).

Neuronal signals were amplified using a MultiClamp 700B amplifier (Molecular Devices, Forster City, CA), low-pass filtered at $1 \mathrm{kHz}$ for voltage clamp recordings, and digitized at $20 \mathrm{kHz}$ using a Digidata $1440 \mathrm{~A}$ interface controlled by 
pClamp 10.6 (Molecular Devices). To record mEPSCs, D-AP5 (50 $\mu \mathrm{M}$, Tocris) and tetrodotoxin (TTX, $1 \mu \mathrm{M}$, Tocris) were added to the ACSF. The electrode internal solution contained (in mM): $130 \mathrm{~K}$-gluconate, $10 \mathrm{HEPES}, 4 \mathrm{KCl}, 4 \mathrm{ATP}$-Mg, 0.3 GTP-Na, $2 \mathrm{NaCl}, 1$ EGTA, and 14 phosphocreatine (pH 7.2, 295-300 mOsm). To record miniature inhibitory postsynaptic currents (mIPSCs), the ACSF contained $1 \mu \mathrm{M}$ tetrodotoxin (TTX) and $10 \mu \mathrm{M}$ CNQX, and the electrode internal solution contained (in mM): $125 \mathrm{KCl}, 2.8 \mathrm{NaCl}, 2 \mathrm{MgCl}_{2}, 0.3 \mathrm{Na}_{3} \mathrm{GTP}, 2 \mathrm{Mg}^{2+}$-ATP, 1 EGTA, 10 HEPES, and 10 phosphocreatine (pH 7.25, 300 mOsm). In some mEPSC/mIPSC recording experiments, 0.15\% biocytin (W/V) was included in the recording electrode to reveal neuronal morphology. During the recording, series resistance (Rs, less than $25 \mathrm{M} \Omega$ ) was constantly monitored and neurons with $>20 \%$ variations in Rs were not included for analyses.

To measure AMPA/NMDA receptor current ratio (A:N) in L5 mPFC neurons and hippocampal CA1 neurons, a bipolar stimulating electrode (FHC, Bowdoin, ME) was placed in L2/3 of the mPFC or on the CA1 input Schaffer collateral fibers. A Cs+-based internal solution (contains in mM, $125 \mathrm{Cs}$-gluconate, $5 \mathrm{TEA}-\mathrm{Cl}, 10 \mathrm{HEPES}, 2.5 \mathrm{CsCl}, 8 \mathrm{NaCl}, 5$ QX314- $\mathrm{HCl}, 4 \mathrm{Mg}^{2+}-\mathrm{ATP}, 0.3 \mathrm{Na}_{3} \mathrm{GTP}, 1$ EGTA and 10 phosphocreatine, pH 7.25) was used. Evoked monosynaptic responses were obtained when neurons were sequentially voltage clamped at -70 mV (for AMPAR-mediated synaptic currents) and $+40 \mathrm{mV}$ (AMPAR+NMDAR currents). A:N ratio was quantified using the peak of EPSC amplitude at -70 $\mathrm{mV}$ ( AMPAR current), and $+40 \mathrm{mV}$ (NMDAR current, which is measured at $75 \mathrm{~ms}$ after stimulus onset ${ }^{21}$ ).

\section{Neuronal morphology}

Analyses of the recorded L5 mFPC neuronal morphology, including dendritic arborization and spine morphometric measures, was performed as described previously ${ }^{22,23}$. After completion of whole cell recording, neurons were injected with 500pA current to facilitate biocytin diffusion into the neurite processes. The slices were then fixed in $4 \%$ PFA overnight, blocked with 1\% BSA/0.01M PBS, permeabilized with 0.2\% Triton X-100. Slices were further incubated with avidin-Alexa 488 for $24 \mathrm{~h}$ and mounted on glass slides with a pair of $\sim 350 \mu \mathrm{m}$ spacers to prevent tissue crushing. Neuronal dendritic arbors were reconstructed after collecting Z-stack images on a confocal microscope (Zeiss LSM 710). The maximal projection images were then imported into FIJI/ImageJ, and neurite arborization and Sholl analysis ${ }^{24}$ were done using the Simple Neurite Tracer plugin ${ }^{25}$. This allows quantification of morphometric features such as dendritic arbor, length, and number of intersections as a function of distances from soma. To analyze spines, Z stacks of spines from the apical dendrites (200-450 $\mu \mathrm{m}$ away from soma, secondary branches) were collected with a 63x objectives (Plan-Apochromat, NA 1.4). Each Z stack was collected at $512 \times 512$ pixels, with $4 \times$ digital zoom and 0.2 $\mu \mathrm{m}$ Z step size. Imaris software (V8.02, Bitplane, South Windsor, CT) was used to measure spine length, density, and head volumes, as we described previously 22.

\section{Laser scanning photostimulation for functional circuit mapping}


To investigate how transgenic mutant APP/PS1 expression affects synaptic connectivity, we used laser scanning photostimulation (LSPS) combined with glutamate uncaging 26,27 to map synaptic connectivity made onto the $L 5$ pyramidal neurons in the mPFC. 5XFAD and littermate control mice were sacrificed at P25-35, during which intracellular APP overload was confirmed by immunohistochemistry staining. Parasagittal mPFC slices were made and perfused in modified ACSF $\left(4 \mathrm{mM} \mathrm{Ca}^{2+}, 4 \mathrm{mM} \mathrm{Mg}^{2+}\right)$ that contains $0.2 \mathrm{mM} \mathrm{MNI-caged} \mathrm{glutamate} \mathrm{and} 5 \mu \mathrm{M}$-CPP (block NMDA receptors and short-term plasticity). To minimize truncation of dendritic structures and preserve connectivity, only L5 neurons with pyramidal shaped soma that are $>50 \mu \mathrm{m}$ below the slice surface were selected for recording/mapping.

LSPS mapping/glutamate uncaging was performed using a 4× objective lens (NA 0.16; Olympus) and a UV laser (355 $\mathrm{nm}$; DPSS Lasers). 1-ms, 20-mW UV laser pulses were delivered onto slices through a pair of $\mathrm{X}-\mathrm{Y}$ mirror to generate a $16 \times 16$ stimulation grid with $100 \mu \mathrm{m}$ spacing. The top of stimulation grid was aligned with the pia surface, and span the pia to white matter. Stimulation location was registered onto a digital image using a CCD camera (Retiga 2000DC, Qimaging). Laser power/timing was controlled by an optic shutter (Conoptics, model 3050), a mechanical shutter (Unibliz VCM-D1) and a neutral density filter (Edmund Optics), and constantly monitored using a photodiode (Edmund Optics) and current amplifier (Sanford Research Systems, model SR570) that feeds to the BNC-6259 boards (National Instruments, Austin, TX). Neuronal signals were amplified with a Multiclamp 700B amplifier, digitized at 10 $\mathrm{kHz}$, and acquired using two BNC-6259 boards. Data synchronization, acquisition and analyses were implemented by Ephus software, a suite of customized MATLAB scripts ${ }^{27}$.

\section{Immunohistochemistry/Immunofluorescence}

Mice were anesthetized with $4 \%$ isoflurane, and transcardially perfused with $0.01 \mathrm{M}$ PBS, followed by cold $4 \%$ paraformaldehyde (PFA) formulated in $0.1 \mathrm{M}$ phosphate buffer ( $\mathrm{pH} 7.4$ ). Brains were post-fixed in cold 4\% PFA overnight at $4^{\circ} \mathrm{C}$, and cryoprotected in $30 \%$ sucrose for $2 \mathrm{~d}$. The brains were then embedded in OCT, and cut into $40-$ $\mu \mathrm{m}$ frozen sections on a sliding microtome (Leica SR-2000). Brain sections were washed three times in 0.01M PBS, and permeabilized in PBS-0.2\% Triton. For sections used for APP/A $\beta$ staining, the free-floating sections were blocked in primary antibody dilution solution ( $0.2 \%$ Triton, $5 \%$ normal goat serum and $1 \%$ bovine serum albumin in $0.01 \mathrm{M}$ PBS) for $2 \mathrm{~h}$, and incubated with anti-APP/A $\beta$ primary antibody (6E10, Biolegend, 1:500 dilution) for 24 , followed by Alexa 555-conjugated goat anti-mouse antibody. For APP/A $\beta$, Iba1 and Thio-S triple staining, sections were similarly blocked in primary antibody dilution solution, and incubated with anti-APP/A $\beta$, anti-lba1 (\#019-19741, Wako, 1:500 dilution) primary antibodies for $24 \mathrm{~h}$. After incubation with A555-conjugated goat anti-mouse and A488-conjugated goat anti-rabbit antibodies for $24 \mathrm{~h}$, sections were extensively washed in 0.01M PBS, and further stained with $0.025 \%$ Thio-S (prepared in 50\% ethanol-50\% PBS) for 10min. These sections were mounted to SuperFrost Plus slides (VWR 
Scientific, West Chester, PA) using DAPI-containing Vectashield mounting medium (H-1200, Vector Laboratories). Images were collected on a LSM 710 confocal microscope (Zeiss) with appropriate laser lines.

\section{Statistical analysis}

All results were reported as mean \pm s.e.m. (standard error of the mean). The experimenters were blinded to mouse genotypes/grouping during data collection and analyses. Sample sizes and number of independent experiments were estimated by power analyses using an R script that takes pre-specified effect size, type I and II errors as input arguments. Sex-segregated data were first analyzed for potential sex-specific effects, and pooled together for group analyses. Male and female data were visualized separately where applicable. Shapiro-Wilk test and $F$ test were first employed to test normality and equal variance. For normal-distributed/equal variance data, Student $t$ test or one/two-way analysis of variations were used. A nonparametric Mann-Whitney $U$ test was used for non-normally distributed/ordinal type data types. Kolmogorov-Smirnov (K-S) test was used to compare cumulative distributions of mEPSC amplitudes. Statistical analyses and graphing were performed using GraphPad Prism 8.0, Microsoft Excel, MATLAB. Figures were prepared using Adobe Creative Cloud. $p<0.05$ was considered statistically significant for all tests.

\section{RESULTS}

\section{APP/A $\beta$ overload in mPFC L5 neuron during early postnatal neurodevelopment}

It has been previously shown that 5XFAD mice exhibit a robust $A \beta$ pathology with an onset of extracellular plaque pathology at approximately 2 months of age, and manifest abundant intraneuronal and plaqueassociated pathology as early as 3 months of age ${ }^{4}$. Because of Thy 1 promoter-driven transgenic APP/PS1 expression is enriched in layer 5 neurons and is across life span ${ }^{12,13}$, we asked whether intraneuronal APP/A $\beta$ load can be detected in cortical L5 neurons at very early developmental stages. We conducted $\mathrm{IHC}$ staining to detect $\mathrm{APP} / \mathrm{A} \beta$ (6E10 antibody), and found that in most cortical regions including the prefrontal cortex, retrosplenial cortex, L5 neurons show strong APP/Ab immunoreactivity at postnatal day (P) 21, which appear stronger than CA1 neuron immunolabeling (Fig 1A). In addition, we observed strong APP/A $\beta$ immunoreactivity in the subiculum region in the temporal levels of hippocampus, consistent with reports that the subiculum is one of earliest structure that shows APP/A $\beta$ overloading ${ }^{4}$. We also found that APP/A $\beta$ overloading in L5 neuron at this early age is associated with few lba1-positive activated microglia (Fig. 1B) that is comparable to the density of Iba1-labeled microglia non-transgenic controls 
(not shown). Importantly, at this early age, no extracellular amyloid plaque or Thio-S+ insoluble fibrillar dense core A $\beta$ deposits was observed. In contrast, mPFC layer 5 neurons in 5 months old 5XFAD mice show extensive APP/A $\beta$ immunoreactivity, increased $A \beta$ plaque deposition, clustered lba- $1+$ reactive microglia surrounding the plaques, and the Thio-S -labeled dense core fibrillar amyloid deposits (Fig 1B). Notably, the APP/A $\beta$ intracellular overloading in L5 mPFC neurons was also seen at earlier ages (P11 and P14, data not shown), indicating transgenic expression of mutant APP/PS1 is a continuous process spanning early neurodevelopment.

\section{Impaired L5 PFC neuron synaptic plasticity early in development}

It has been well established that impaired plasticity, such as LTP, is a core synaptic deficit in AD brain 28,29 . Because of the continuous APP/A $\beta$ intracellular overloading in L5 neurons from very early age, and previous reports that intraneuronal $A \beta$ accumulation being detectable already at the age of 6 weeks ${ }^{4}$, we reason that $\mathrm{mPFC}$ L5 neuron may exhibit a differential susceptibility to plasticity disturbances compared to other brain structures, such as the hippocampus. We recorded LTP in both mPFC and hippocampus slices in 6-8 wks 5XFAD mice, and found that mPFC L5 LTP, induced by tetanus stimulations, were significantly impaired at this age (Fig 2A. Averaged LTP amplitude during the last $10 \mathrm{~min}$ of post-stimulation recordings: $\left.\mathrm{WT}, 165.4 \pm 1.24 ; 5 \mathrm{XFAD}, 136.0 \pm 1.21, \mathrm{t}_{(13)}=16.9, \mathrm{p}<0.0001\right)$. In comparison, there existed no difference in the LTP amplitudes in CA1 (Fig. 2C. WT, $170.8 \pm 1.90 ; 5 X F A D, 168.0 \pm 1.42, \mathrm{t}_{(16)}=1.11, \mathrm{p}=$ 0.28). To ask whether the impaired MPFC L5 LTP is age-dependent, we recorded LTP at an earlier stage (P22-30), and found that no difference between 5XFAD and control brain (Fig. 2B. WT, $171.1 \pm 1.89$; 5XFAD, $\left.174.0 \pm 1.66, t_{(14)}=1.15, p=0.27\right)$. These data suggest that impaired mPFC LTP likely develops as a result of increased age-dependent intracellular APP overload, which is consistent with the observation that CA1 LTP impairment is observed at 5-6 months old mice (Fig. 2D. WT, $161.1 \pm 1.97 ; 5 X F A D, 115.7 \pm$ $\left.1.14, t_{(14)}=21.0, p<0.0001\right)$, during which $A \beta$-relat ed pathology in hippocampus is well developed ${ }^{4}$.

\section{Impaired synaptic transmission in L5 PFC neuron at early postweaning age}

Because of the increased intracellular APP/A $\beta$ overloading was observed at P21, we asked whether synaptic activity is affected at this early post-weaning age. We conducted patch clamp recordings of mEPSCs in both mPFC L5 pyramidal neurons and hippocampal CA1 neurons. Recordings from L5 mPFC neurons (Fig. 3A) revealed that 5XFAD mice show reduced mEPSC amplitude (Fig. 3B. WT, $n=1244$ events $/ 8$ cells $/ 5$ mice; 5 XFAD, $n=1165$ events $/ 6$ cells $/ 6$ mice; $D=0.14, K-S$ test, $p<0.0001$ ) and frequency (Fig. 3C. Events/sec: WT, $3.72 \pm 0.38 ; 5 X F A D, 2.48 \pm 0.28, p=0.018$ ). In addition, 5xFAD neurons showed a significantly reduced AMPA/NMDA receptor current ratio (Fig. 3D. WT, $1.43 \pm 0.06$; 
SXFAD, $\left.1.16 \pm 0.07 ; \mathrm{t}_{(18)}=2.97\right)$, indicating impairment of synaptic transmission or synapse maturation. In comparison to L5 mPFC neurons, CA1 neuron of the same age did not show alterations of mEPSC amplitude distribution (Fig. 3E, F. WT, $\mathrm{n}=765$ events $/ 5$ cells $/ 4$ mice; 5XFAD, $n=1103$ events $/ 7$ cells $/ 6$ mice; $D=0.057, \mathrm{~K}-\mathrm{S}$ test, $\mathrm{p}=0.105$ ), nor was there change in mEPSC frequency (Fig. 3G. Events/sec, WT, $2.05 \pm 0.25 ; 5 X F A D, 1.98 \pm 0.28, p=0.85)$. We also did not observe significant change on the AMPA/NMDA current ratio in CA1 neurons (Fig. 3H. WT, $1.45 \pm 0.06 ; 5 X F A D, 1.44 \pm 0.07, p=0.93$ ). These data suggest altered synaptic activity selectively occur at L5 mPFC neurons at an very early developmental stage, potentially as a result of the detrimental effect of increased APP/ A $\beta$ overloading in mPFC L5 neurons early in development.

Decreased dendritic spine size and density in L5 mPFC neurons in early postweaning 5XFAD mice

We next focused on potential anatomical changes of MPFC L5 neurons during early development by analyzing the dendritic structure and spine morphometry at P22-30, during which we found impaired synaptic activity. L5 neuron morphology was revealed by biocytin injection (Fig. 4A), followed by confocal imaging of the dendritic structure and Z-stack images of spines. Sholl analysis revealed that 5XFAD did not affect the number of dendritic intersections as a function of distance from soma (Fig. 4B. Main group effect: $F_{(1,15)}=2.93, p=0.11$, two-way ANOVA), nor did it affect dendritic length distribution (Fig. 4C. main group effect: $F_{(1,15)}=0.033, p=0.86$, two-way ANOVA). However, dendritic spine from 5XFAD neurons exhibited a reduced density (Fig. 4D, 4E. Number of spines/10 $\mu \mathrm{m}$ : WT, $12.52 \pm 0.45 ; 5 X F A D, 10.8$ $\pm 0.37, \mathrm{t}_{(21)}=2.89, \mathrm{p}=0.008$ ). In comparison, spine length from 5XFAD L5 neurons did not differ (Fig. $\mathbf{4 F}$. Averaged length in $\mu \mathrm{m}$ : WT, $\left.2.06 \pm 0.13 ; 5 X F A D, 2.20 \pm 0.17, t_{(21)}=0.69, p=0.49\right)$. We next quantified the spine head volume, and found that 5XFAD neurons show significantly reduced spine head volume distribution (Fig. 4G. WT, $\mathrm{n}=274$ spines $/ 6$ neurons $/ 6$ mice; $5 X F A D, n=258$ spines $/ 7$ neurons $/ 5$ mice. $D=$ $0.286, p<0.0001, \mathrm{~K}-\mathrm{S}$ test). Considering that spine size and geometry are well established to correlate with glutamate receptor content and functional maturity ${ }^{30}$, these data are consistent with decreased mEPSC amplitude and frequency, and further suggest impaired synaptic function at very early development age in the PFC.

mPFC L5 neurons from 5XFAD mice show reduced intracortical circuit connectivity at 4-5 wks age

We hypothesize that the early transgenic mutant APP/PS1 expression may alter intracortical functional connectivity in L5 mPFC neurons, either as a result of impaired establishment of developmental circuits, or 
as a result of early degradation of synaptic connectivity. mPFC cortical circuits show conserved connectivity patterns, with balanced excitation and inhibition distributed across both columnar and laminar dimensions $26,31,32$. We used LSPS mapping ${ }^{14,26,27}$ to investigate synaptic connectivity made onto L5 pyramidal neurons in MPFC in parasagittal brain slices (Fig. 5A). L5 neurons from P25-35 5XFAD mice and littermate controls were voltage clamped, and glutamate uncaging at different cortical locations produces excitatory currents that reflect either soma/dendrite-mediated direct EPSC responses or synaptic EPSC responses (see methods) (Fig. 5B). In addition, by holding at $0 \mathrm{mV}$, postsynaptic inhibitory currents (IPSCs) can be also obtained. This allows us to construct a 'map' of local circuit connectivity (both excitatory and inhibitory. Fig. 5C).

We compared both excitatory and inhibitory inputs onto L5 mPFC pyramidal neurons from WT and 5XFAD groups after collecting many cells/animals. As expected, L5 neurons receive primary inputs from L2/3 (Fig. 5D). When the strength of this connectivity was quantified, we found that overall connectivity pattern, reflected by averaged synaptic current distribution across binned cortical depth, was dramatically reduced (Fig. 5E. Main effect of group, $F_{(1,22)}=14.8, p=0.0009$. Two-way ANOVA with repeated measures). In addition, combined L2/3 inputs in 5XFAD neurons were significantly reduced (Fig. 5F. WT, $56.4 \pm 4.3$ pA; 5XFAD, $\left.33.5 \pm 2.8 p A . t_{(22)}=4.48, p=0.0002\right)$. This reduction of connectivity was seen only for excitatory connectivity, as overall inhibitory connectivity pattern did not change (Fig. 5G, 5H. Main effect of group, $F_{(1,24)}=0.97, p=0.48$. two-way ANOVA with repeated measures). The combined inhibitory inputs from L2/3 and L5 also did not change (Fig. 5I. WT, $37.9 \pm 1.9$ pA; 5XFAD, $39.7 \pm 2.3 p A . t_{(24)}=0.59, p=0.56$ ). These data suggest reduced excitatory synaptic connectivity onto L5 neurons in mPFC at very early (4-5 wks) age, and are consistent with reduced mEPSC amplitude/frequency and dendritic spine size and density found on these neurons.

\section{DISCUSSION}

In this study, we report very early synaptic deficits in 5XFAD mice, which include impaired synaptic plasticity (LTP) in the mPFC L5 neurons, deficits in synaptic activity and intracortical connectivity. These functional changes are correlated with morphological alternations at dendritic spine levels and reflect the age-dependent intracellular transgenic APP/A $\beta$ overloading. Our data support that 5XFAD transgenic expression of mutant APP/PS1 leads to impaired development of neuronal function and connectivity at very early post-weaning age. The main focus of this paper is not to exhaustively pursue age-dependent pathological or electrophysiological changes, but rather to focus on L5 mPFC neurons at a very early age 
and probe circuit impairment using sensitive physiological and morphological measures. To the best of our knowledge, this study reports the earliest synaptic function and connectivity changes in 5XFAD mice in a major mPFC projection neuron population. Our data demonstrate that mutant forms of APP/PS1 and associated $A \beta$ production could pose a neurodevelopmental syndrome featuring early synaptic loss and dysconnectivity. While it is possible that by early post-weaning ages, synaptic loss and degradation already occurs as a result of APP/A $\beta$ overloading, an alternative explanation for these observations is that the synaptic connectivity, maturation, and circuit function in L5 mPFC neurons in 5XFAD mice are impaired developmentally and never achieved the same functionality as the control neurons.

Using immunohistochemistry staining, we have shown that L5 mPFC neurons exhibit increased intracellular APP/A $\beta$ loading at P21 and earlier. Similarly, another vulnerable cortical population in terms of $A P P / A \beta$ overloading is the subiculum neurons of the temporal hippocampus, whose functional changes remain to be investigated. Because the $6 \mathrm{E} 10$ antibody for $A \beta$ does not distinguish APP and $A \beta^{33,34}$, we cannot attribute these observed synaptic and circuit connectivity changes to APP or to intracellular A $\beta$ production. Yet, our IHC staining revealed that minimum microglia activation at P21, and there was no extracellular amyloid deposition or dense core fibrillar $A \beta$ plaques in extracellular space. It is therefore less likely L5 neuronal loss occurs at this early age. It has been reported that cortical L5 neurons are among the first neuronal populations that develop synaptic pathology 4,7 , including decreased spontaneous mEPSCs frequency and amplitude, higher threshold for firing of action potentials, which happened before any overt structural dystrophy ${ }^{7}$. Yet, these pathological changes occur at much later stages that those observed by this study.

It is unclear how mutant APP over-expression affects synaptic function in the developing brain and how these detrimental effects evolve with age. Neurophysiological data exploring the effects of transgenic APP/PS1 on the prefrontal cortex circuit function are rather limited. Yet, mPFC is a primary and early target that develops AD pathology ${ }^{35-37}$. Transgenic overloading of APP/A $\beta$ in the developing cortical circuits may disrupt a myriad physiological function of neurons, including defective endo-lysosomal trafficking ${ }^{38}$ 39, impaired intracellular cargo transport ${ }^{40}$, altered intracellular signaling ${ }^{41,42}$, or defective neurotransmitter release ${ }^{43}$ that collectively contribute to failure of synaptic connectivity or synaptic degradation. That the impaired synaptic plasticity/LTP was observed at 6-8 wks, but not at early postweaning (P22-30), during which synaptic connectivity was reduced, suggest disruption of plasticityrelated mechanisms by $A P P / A \beta$ may not be as sensitive as connectivity of developing cortical circuits. In 
some studies impaired LTP has been detected in AD mice models before 4 months of age ${ }^{44,45}$ but typically appears at later age when intracellular $A \beta$ load in higher ${ }^{6,46-51}$. It was also reported that LTP in layer 5 of somatosensory cortex was more severely impaired than LTP triggered in CA1 area in 6 months 5xFAD mice, which could be attributed to increased somatosensory cortex amyloid deposition ${ }^{52}$. It has also been reported that theta burst stimulation-induced hippocampus LTP is impaired at 6 months but not prior to 4 months ${ }^{6,52}$. In contrast to these reports, using a stronger LTP induction protocol (tetanus stimulation), we found that LTP in L5 mPFC neurons was impaired as early as 6-8 wks, during which the CA1 LTP seems normal. This may be due to overall higher L5 mPFC neuron intracellular APP/ A $\beta$ production or increased vulnerability of this neuronal population.

In summary, our study revealed that synaptic function and plasticity changes occur at very early postweaning age in the 5XFAD AD mouse model, and provided the first demonstration of reduced circuit connectivity in a major prefrontal projection neuron type as a result of APP/A $\beta$ overloading. These functional disruptions suggest that transgenic mutant APP/PS1 over-expression has a profound effect on developing cortical circuit, and as such changes in neuronal function may be a life-long process that impact neuronal degeneration in later stages. This thought-provoking hypothesis justifies further experimentation.

\section{FIGURE LEGEND}

FIGURE 1. Age-dependent transgenic APP/A $\beta$ overloading and pathology in the $m P F C$. (A) IHC using 6E10 antibody revealed extensive L5 intraneuronal labeling of APP/A $\beta$ in prefrontal cortex (left) and to a lesser extent, in the retrosplenium cortex. CA1 neurons also show relatively weaker APP/A $\beta$ immunoreactivity. In comparison, hippocampus subiculum and CA1 neurons on the temporal levels show stronger APP/A $\beta$ immunoreactivity. (B) Triple IHC staining of APP/A $\beta$, Iba1 and Thio-S to label amyloid deposition, reactive microglia and dense core fibrilliar amyloid plaques. L5 neurons of P21 mPFC show strong intracellular APP/A $\beta$ labeling, with minimum reactive microglia and no Thio-S+ plaque deposition. In contrast, L5 neurons from P150 mPFC show strong extracellular amyloid deposition with dense Thio-S+ fibriliar A $\beta$ cores that are surrounded by large numbers of reactive microglia.

FIGURE 2. Age-dependent impairment of LTP in the mPFC and hippocampus. (A) In P42-56 5XFAD and wild type control mice, LTP magnitude in MPFC L5 regions, measured as the averaged percent of increase of fEPFP slope, is significantly lower in 5XFAD mice. $* * * p<0.0001$. (B) LTP magnitude does not differ at an earlier age (P22-30) in the mPFC. (C) Similar levels of LTP magnitude was observed during the last $10 \mathrm{~min}$ of the hour-long 
LTP recordings. $\mathrm{p}=0.28$. Markers: grey filled, male; open circle, female. (D) Impaired LTP in 5XFAD hippocampus slices was observed in 5-6 months mice. $* * * * p<0.0001$.

FIGURE 3. Reduced mEPSC amplitude and frequency are selectively observed in developing L5 mPFC neurons at a very early stage. (A) Representative whole cell patch clamp recording of spontaneous miniature excitatory postsynaptic currents for both WT and 5XFAD neurons. (B) Quantification of mEPSC events. mEPSC amplitudes from 5XFAD neurons were clustered to the left. There was significant difference between the cumulative distribution curve (K-S test, $* * * * p<0.0001)$. Inset, violin plot of all the pooled mEPSC amplitude. (C) L5 5XFAD neurons show reduced mEPSC frequency $(* p=0.018$ ). (D) L5 5XFAD neurons exhibit significantly reduced AMPA/NMDA receptor current ratio $(* * \mathrm{p}=0.008)$. (E) ) Whole cell recording of spontaneous mEPSCs from both WT and 5XFAD hippocampus CA1 neurons. (F) No significant difference of binned amplitude distribution was observed ( $\mathrm{p}=0.105$, K-S test). (G) No significant changes on mEPSC frequency in CA1 neurons between WT and 5XFAD neurons $(\mathrm{p}=0.85)$. $(\mathrm{H})$ AMPA/NMDA receptor current ratio does not statistically differ $(\mathrm{p}=0.93)$.

FIGURE 4. Reduced dendritic spine size and density in L5 mPFC at early post weaning age. (A) Morphology reconstruction of L5 mPFC neurons. (B) Sholl analysis of representative L5 neurons dendritic arbor in both WT and 5XFAD neurons. No statistical effects on genotype was observed $(\mathrm{p}=0.107$ for genotype effect, two-way ANOVA. (C) Sholl analyses on dendritic length distribution. No significant difference exists for the dendritic length as a function of distance from soma between WT and $\operatorname{XFAD}$ neurons $\left(\mathrm{F}_{(1,15)}=0.033 ; \mathrm{p}=0.86\right.$ for main group effects). (D) Representative dendritic spines from WT and 5XFAD mPFC L5 pyramidal neuron apical dendrites. (E) 5XFAD neurons show decreased spine density $(* * \mathrm{p}=0.008)$. (F) 5XFAD neurons do not differ in spine length $(\mathrm{p}=0.49)$. (G) Cumulative distribution of spine head volume. Spine head volume from 5XFAD neurons show significantly reduced volume distribution $(* * * \mathrm{p}<0.001, \mathrm{~K}-\mathrm{S}$ test). Inset, box-whisker plot of pooled spine head volumes.

FIGURE 5. mPFC L5 neurons from $5 X F A D$ mice show reduced intracortical synaptic connectivity at early postweaning age (P25-35). (A) Schematic illustration of a parasagittal mPFC slice preparation, with whole cell recording and LSPS mapping on an L5 pyramidal neuron. A $16 \times 16$ stimulus grid was centered on mPFC and aligned with pia surface. Cyan asterisks indicate glutamate uncaging locations. (B) LSPS mapping/glutamate uncaging at different locations elicit direct soma responses, excitatory synaptic responses (EPSC), inhibitory synaptic response (IPSC), or no response. (C) Illustration of LSPS mapping, with representative mapping traces of a $8 \times 8$ array of excitatory and inhibitory responses. Triangle indicate soma location. (D) Representative averaged excitatory response maps from WT $(\mathrm{n}=12$ neurons $/ 7$ mice $)$ and 5XFAD neurons $(\mathrm{n}=12$ neurons $/ 8$ mice $)$. Triangle denotes soma location. (E) Averaged strength of synaptic inputs binned by cortical layers. 5XFAD neurons show significantly altered laminar inputs $(* * * p=0.0009$ for genotype effect. Two-way ANOVA; $\# p<0.05$, Sidak's multiple comparison test). (F) Combined L2/3 inputs from 5XFAD neuron show significant reduction in connectivity strength (***p $=0.0002)$. $(\mathrm{G})$ No change on overall inhibitory synaptic inputs across binned cortical depth ( $p=0.48$. Two-way ANOVA). (F) Combined L23 and L5 inhibitory synaptic inputs from 5XFAD neuron show no change compared with those from control neurons $(\mathrm{p}=0.56)$. 


\section{REFERECE}

1. De-Paula VJ, Radanovic M, Diniz BS, Forlenza OV. Alzheimer's disease. Sub-cellular biochemistry 2012;65:329-352.

2. Soria Lopez JA, González HM, Léger GC. Alzheimer's disease. Handbook of clinical neurology 2019;167:231-255.

3. Duyckaerts C, Potier MC, Delatour B. Alzheimer disease models and human neuropathology: similarities and differences. Acta neuropathologica 2008;115:5-38.

4. Oakley H, Cole SL, Logan S, et al. Intraneuronal beta-amyloid aggregates, neurodegeneration, and neuron loss in transgenic mice with five familial Alzheimer's disease mutations: potential factors in amyloid plaque formation. The Journal of neuroscience : the official journal of the Society for Neuroscience 2006;26:10129-10140.

5. Oh SJ, Lee HJ, Kang KJ, et al. Early Detection of Abeta Deposition in the 5xFAD Mouse by Amyloid PET. Contrast Media Mol Imaging 2018;2018:5272014.

6. Kimura R, Ohno M. Impairments in remote memory stabilization precede hippocampal synaptic and cognitive failures in 5XFAD Alzheimer mouse model. Neurobiology of disease 2009;33:229-235.

7. Buskila Y, Crowe SE, Ellis-Davies GC. Synaptic deficits in layer 5 neurons precede overt structural decay in 5xFAD mice. Neuroscience 2013;254:152-159.

8. Seo J, Kritskiy O, Watson LA, et al. Inhibition of p25/Cdk5 Attenuates Tauopathy in Mouse and iPSC Models of Frontotemporal Dementia. The Journal of neuroscience : the official journal of the Society for Neuroscience 2017;37:9917-9924.

9. Madabhushi R, Pan L, Tsai LH. DNA damage and its links to neurodegeneration. Neuron 2014;83:266-282.

10. Martorell AJ, Paulson AL, Suk HJ, et al. Multi-sensory Gamma Stimulation Ameliorates Alzheimer's-Associated Pathology and Improves Cognition. Cell 2019;177:256-271 e222.

11. Seo J, Giusti-Rodriguez P, Zhou Y, et al. Activity-dependent p25 generation regulates synaptic plasticity and Abeta-induced cognitive impairment. Cell 2014;157:486-498.

12. Feng $\mathrm{G}$, Mellor $\mathrm{RH}$, Bernstein $\mathrm{M}$, et al. Imaging neuronal subsets in transgenic mice expressing multiple spectral variants of GFP. Neuron 2000;28:41-51.

13. Bradley JE, Ramirez G, Hagood JS. Roles and regulation of Thy-1, a context-dependent modulator of cell phenotype. BioFactors (Oxford, England) 2009;35:258-265.

14. Qiu S, Anderson CT, Levitt P, Shepherd GM. Circuit-specific intracortical hyperconnectivity in mice with deletion of the autism-associated Met receptor tyrosine kinase. The Journal of neuroscience : the official journal of the Society for Neuroscience 2011;31:5855-5864.

15. Cui $Y$, Jin J, Zhang $X$, et al. Forebrain NR2B overexpression facilitating the prefrontal cortex longterm potentiation and enhancing working memory function in mice. PloS one 2011;6:e20312.

16. Villers A, Ris L. Improved preparation and preservation of hippocampal mouse slices for a very stable and reproducible recording of long-term potentiation. Journal of visualized experiments : JoVE 2013.

17. Larson J, Munkacsy E. Theta-burst LTP. Brain research 2015;1621:38-50.

18. Kenney J, Manahan-Vaughan D. Learning-facilitated synaptic plasticity occurs in the intermediate hippocampus in association with spatial learning. Frontiers in synaptic neuroscience 2013;5:10.

19. Zhang $\mathrm{M}$, Storm DR, Wang H. Bidirectional synaptic plasticity and spatial memory flexibility require $\mathrm{Ca} 2+-$ stimulated adenylyl cyclases. The Journal of neuroscience : the official journal of the Society for Neuroscience 2011;31:10174-10183. 
20. Qiu S, Korwek KM, Weeber EJ. A fresh look at an ancient receptor family: emerging roles for low density lipoprotein receptors in synaptic plasticity and memory formation. Neurobiology of learning and memory 2006;85:16-29.

21. Terashima A, Pelkey KA, Rah JC, et al. An essential role for PICK1 in NMDA receptor-dependent bidirectional synaptic plasticity. Neuron 2008;57:872-882.

22. Peng Y, Lu Z, Li G, et al. The autism-associated MET receptor tyrosine kinase engages early neuronal growth mechanism and controls glutamatergic circuits development in the forebrain. Molecular psychiatry 2016;21:925-935.

23. Qiu S, Lu Z, Levitt P. MET receptor tyrosine kinase controls dendritic complexity, spine morphogenesis, and glutamatergic synapse maturation in the hippocampus. The Journal of neuroscience : the official journal of the Society for Neuroscience 2014;34:16166-16179.

24. Sholl DA. Dendritic organization in the neurons of the visual and motor cortices of the cat. Journal of anatomy 1953;87:387-406.

25. Longair MH, Baker DA, Armstrong JD. Simple Neurite Tracer: open source software for reconstruction, visualization and analysis of neuronal processes. Bioinformatics (Oxford, England) 2011;27:2453-2454.

26. Shepherd GM, Svoboda K. Laminar and columnar organization of ascending excitatory projections to layer $2 / 3$ pyramidal neurons in rat barrel cortex. The Journal of neuroscience : the official journal of the Society for Neuroscience 2005;25:5670-5679.

27. Suter BA, O'Connor T, lyer V, et al. Ephus: multipurpose data acquisition software for neuroscience experiments. Frontiers in neural circuits 2010;4:100.

28. Walsh DM, Klyubin I, Fadeeva JV, et al. Naturally secreted oligomers of amyloid beta protein potently inhibit hippocampal long-term potentiation in vivo. Nature 2002;416:535-539.

29. Shankar GM, Li S, Mehta TH, et al. Amyloid-beta protein dimers isolated directly from Alzheimer's brains impair synaptic plasticity and memory. Nature medicine 2008;14:837-842.

30. Matsuzaki M, Ellis-Davies GC, Nemoto T, Miyashita $Y$, lino M, Kasai H. Dendritic spine geometry is critical for AMPA receptor expression in hippocampal CA1 pyramidal neurons. Nature neuroscience 2001;4:1086-1092.

31. Wood L, Gray NW, Zhou Z, Greenberg ME, Shepherd GM. Synaptic circuit abnormalities of motor-frontal layer $2 / 3$ pyramidal neurons in an RNA interference model of methyl-CpG-binding protein 2 deficiency. The Journal of neuroscience : the official journal of the Society for Neuroscience 2009;29:12440-12448.

32. Xu X, Olivas ND, Ikrar T, et al. Primary visual cortex shows laminar-specific and balanced circuit organization of excitatory and inhibitory synaptic connectivity. The Journal of physiology 2016;594:18911910.

33. Huwait EA, Baghallab IM, Glabe CG, Abulnaja KO, Kumosani TA, Moselhy SS. Identification of amyloid antibodies for Alzheimer disease - immunotherapy. Archives of physiology and biochemistry 2020:1-8.

34. Droste $P$, Frenzel $A$, Steinwand $M$, et al. Structural differences of amyloid- $\beta$ fibrils revealed by antibodies from phage display. BMC biotechnology 2015;15:57.

35. Xu P, Chen A, Li Y, Xing X, Lu H. Medial prefrontal cortex in neurological diseases. Physiological genomics 2019;51:432-442.

36. Bazzigaluppi P, Beckett TL, Koletar MM, et al. Early-stage attenuation of phase-amplitude coupling in the hippocampus and medial prefrontal cortex in a transgenic rat model of Alzheimer's disease. Journal of neurochemistry 2018;144:669-679.

37. Har-Paz I, Roisman N, Michaelson DM, Moran A. Extra-Hippocampal Learning Deficits in Young Apolipoprotein E4 Mice and Their Synaptic Underpinning. Journal of Alzheimer's disease : JAD 2019;72:71-82. 
38. Kepp KP. Alzheimer's disease due to loss of function: A new synthesis of the available data. Progress in neurobiology 2016;143:36-60.

39. Maulik M, Peake K, Chung J, Wang Y, Vance JE, Kar S. APP overexpression in the absence of NPC1 exacerbates metabolism of amyloidogenic proteins of Alzheimer's disease. Human molecular genetics 2015;24:7132-7150.

40. Bhalla A, Vetanovetz CP, Morel E, Chamoun Z, Di Paolo G, Small SA. The location and trafficking routes of the neuronal retromer and its role in amyloid precursor protein transport. Neurobiology of disease 2012;47:126-134.

41. Xie CW. Calcium-regulated signaling pathways: role in amyloid beta-induced synaptic dysfunction. Neuromolecular medicine 2004;6:53-64.

42. Woods NK, Padmanabhan J. Neuronal calcium signaling and Alzheimer's disease. Advances in experimental medicine and biology 2012;740:1193-1217.

43. Ari C, Borysov SI, Wu J, Padmanabhan J, Potter H. Alzheimer amyloid beta inhibition of Eg5/kinesin 5 reduces neurotrophin and/or transmitter receptor function. Neurobiology of aging 2014;35:1839-1849.

44. Ma T, Hoeffer CA, Capetillo-Zarate E, et al. Dysregulation of the mTOR pathway mediates impairment of synaptic plasticity in a mouse model of Alzheimer's disease. PloS one 2010;5.

45. Larson J, Lynch G, Games D, Seubert P. Alterations in synaptic transmission and long-term potentiation in hippocampal slices from young and aged PDAPP mice. Brain research 1999;840:23-35.

46. Fitzjohn SM, Morton RA, Kuenzi F, et al. Age-related impairment of synaptic transmission but normal long-term potentiation in transgenic mice that overexpress the human APP695SWE mutant form of amyloid precursor protein. The Journal of neuroscience : the official journal of the Society for Neuroscience 2001;21:4691-4698.

47. Chapman PF, White GL, Jones MW, et al. Impaired synaptic plasticity and learning in aged amyloid precursor protein transgenic mice. Nature neuroscience 1999;2:271-276.

48. Oddo S, Caccamo A, Shepherd JD, et al. Triple-transgenic model of Alzheimer's disease with plaques and tangles: intracellular Abeta and synaptic dysfunction. Neuron 2003;39:409-421.

49. Kimura R, Devi L, Ohno M. Partial reduction of BACE1 improves synaptic plasticity, recent and remote memories in Alzheimer's disease transgenic mice. Journal of neurochemistry 2010;113:248-261.

50. Hsia AY, Masliah E, McConlogue L, et al. Plaque-independent disruption of neural circuits in Alzheimer's disease mouse models. Proceedings of the National Academy of Sciences of the United States of America 1999;96:3228-3233.

51. Moechars D, Dewachter I, Lorent K, et al. Early phenotypic changes in transgenic mice that overexpress different mutants of amyloid precursor protein in brain. The Journal of biological chemistry 1999;274:6483-6492.

52. Crouzin N, Baranger K, Cavalier M, et al. Area-specific alterations of synaptic plasticity in the 5XFAD mouse model of Alzheimer's disease: dissociation between somatosensory cortex and hippocampus. PloS one 2013;8:e74667. 


\section{Consent for publication}

The manuscript has not been previously published. The authors agree to publication in the journal of Translational Neurodegeneration

\section{Data Availability Statement}

The data used to support the findings of this study are available from the corresponding author upon request.

\section{Conflict of interests}

The authors declare no conflict of interests.

\section{Acknowledgements}

This work was supported by grant from the National Natural Science Foundation of China (No. 81822013).

\section{Authors' Contribution}

Chang Chen: Conceptualization, Formal analysis, Investigation, Writing- main manuscript. Jing Wei:

Conceptualization, Investigation. Xiaokuang Ma: Investigation, Formal analysis. Le Zhang Data Curation, Formal analysis. Antione Nehme: Formal analysis, Visualization, Methodology. Yuehua Cui: Investigation, Data Curation, Formal analysis. Deveroux Ferguson: Investigation, Supervision. Shenfeng Qiu: Conceptualization, Supervision, Formal analysis, Resources, Writing- main manuscript. Feng Bai: Conceptualization, Supervision, Funding acquisition, Writing- main manuscript. 


\section{FIGURES}

Figure 1.
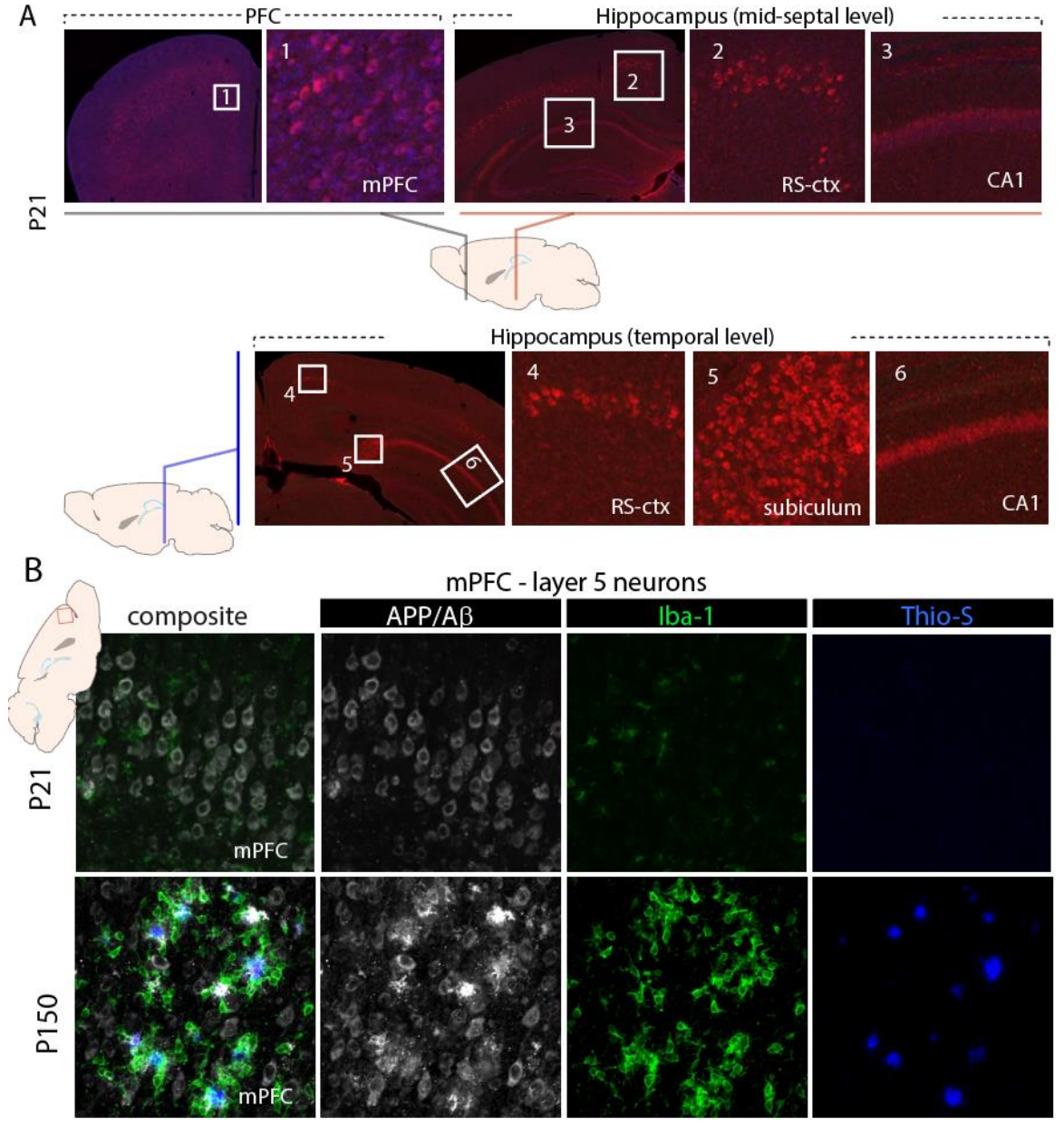
Figure 2.

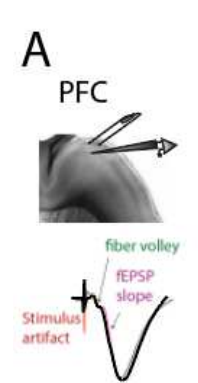

Impaired PFC-LTP at P42-56

Normal PFC-LTP post weaning P22-30
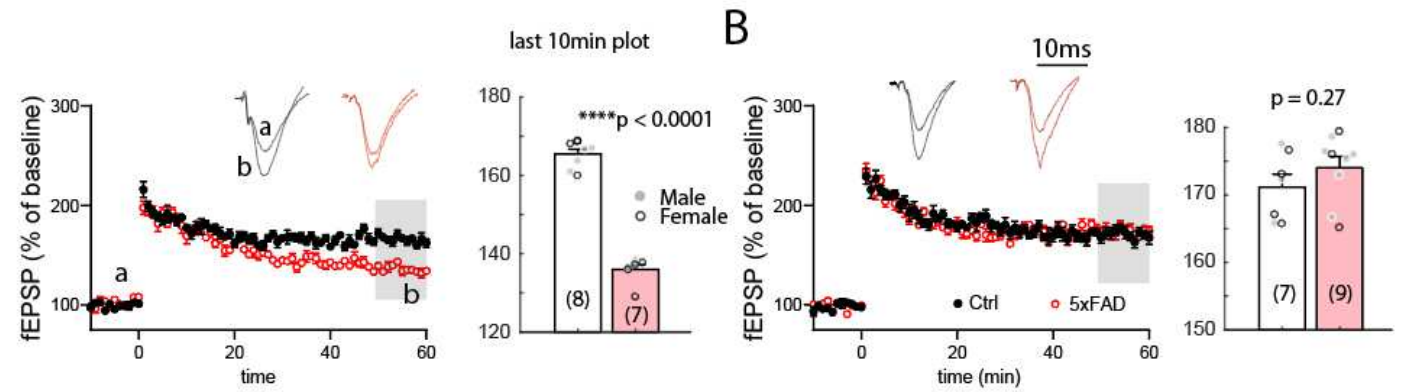

C normal HPC- LTP at P42-56

D Impaired HPC - LTP at 5-6 months

HPC
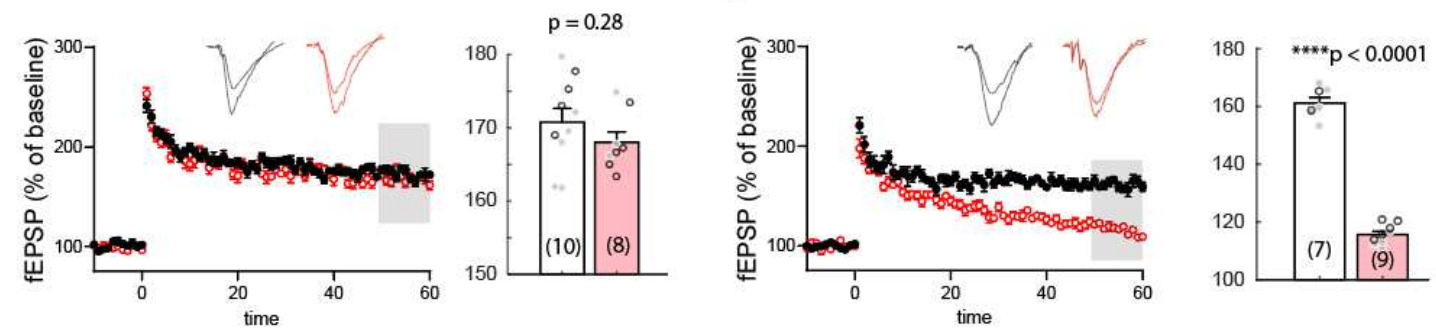
Figure 3.

\section{L5 mPFC projection neurons}
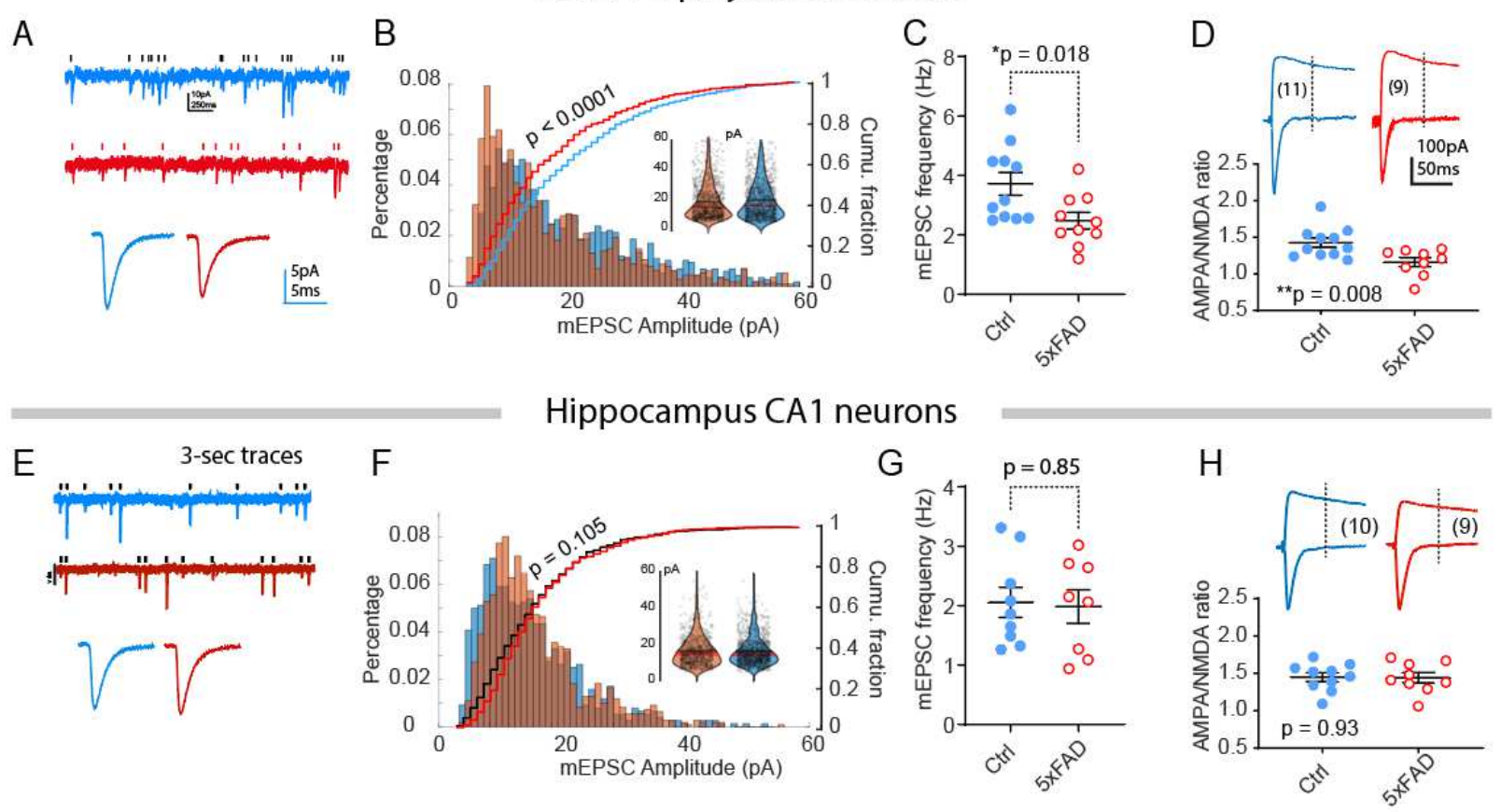
Figure 4 .

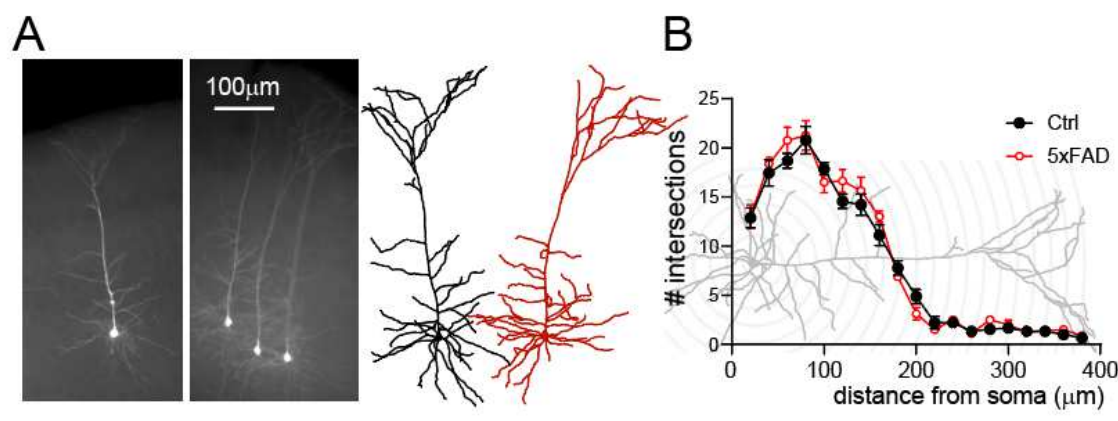

C
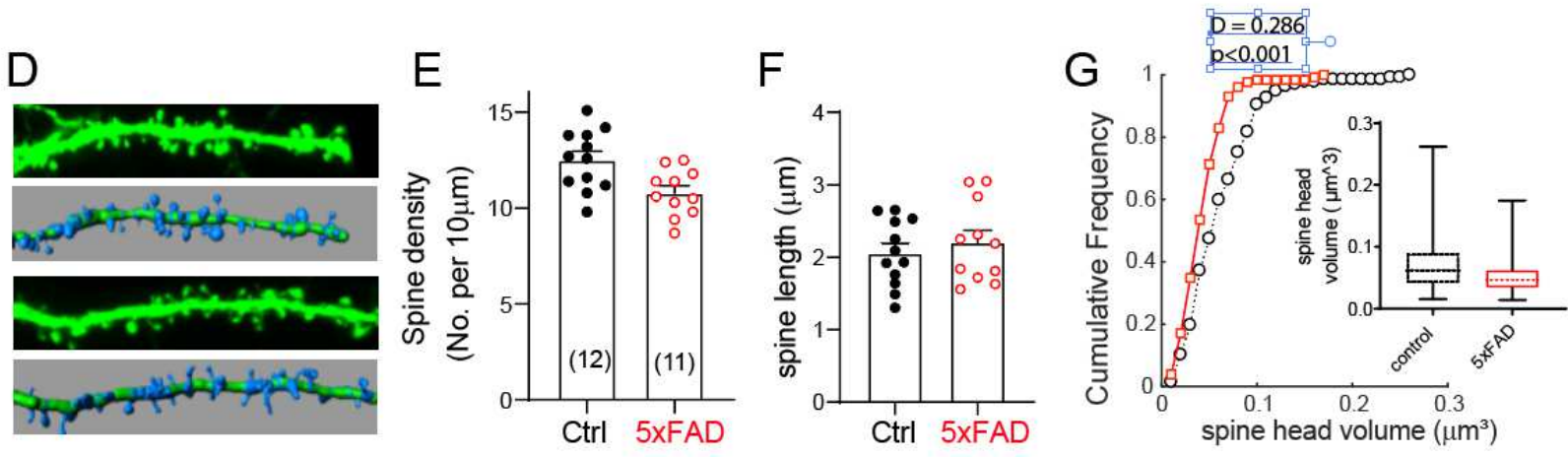
Figure 5.

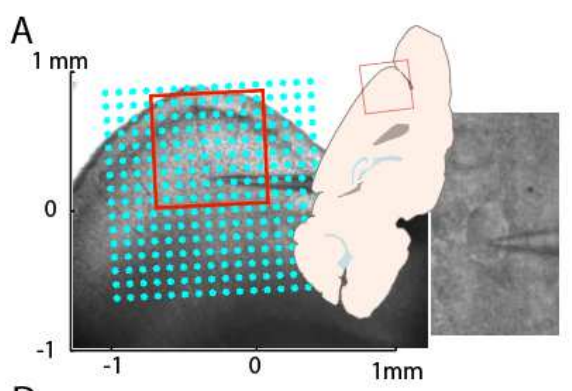

D

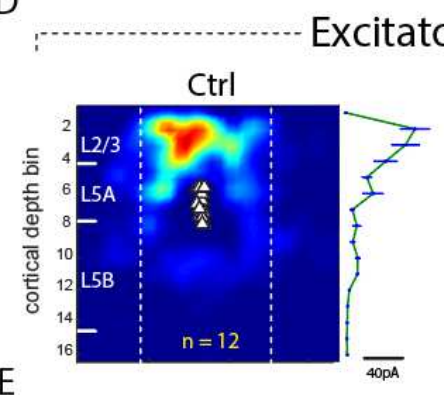

E

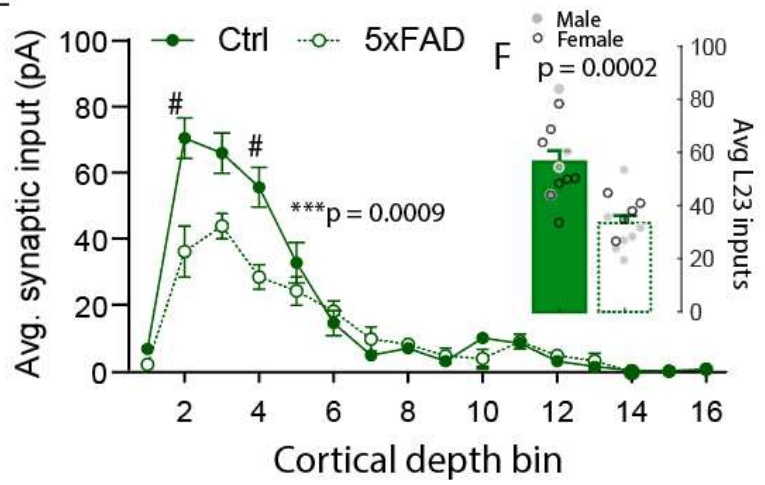

B

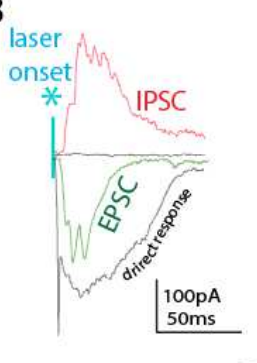

G
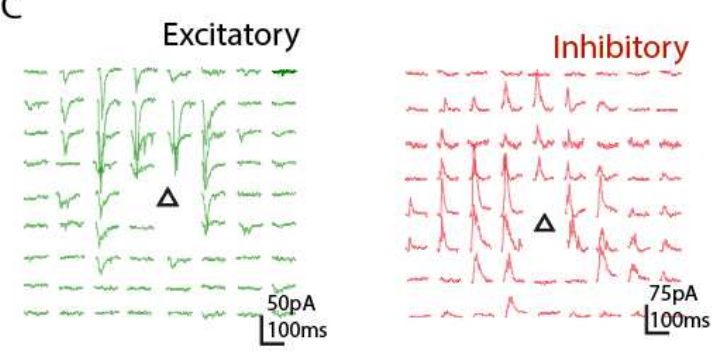

Inhibitory

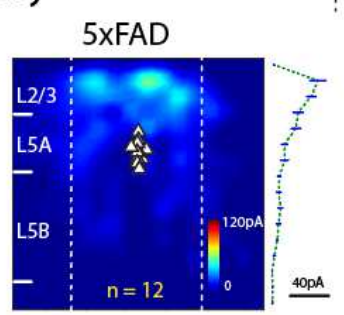

$\mathrm{H}$
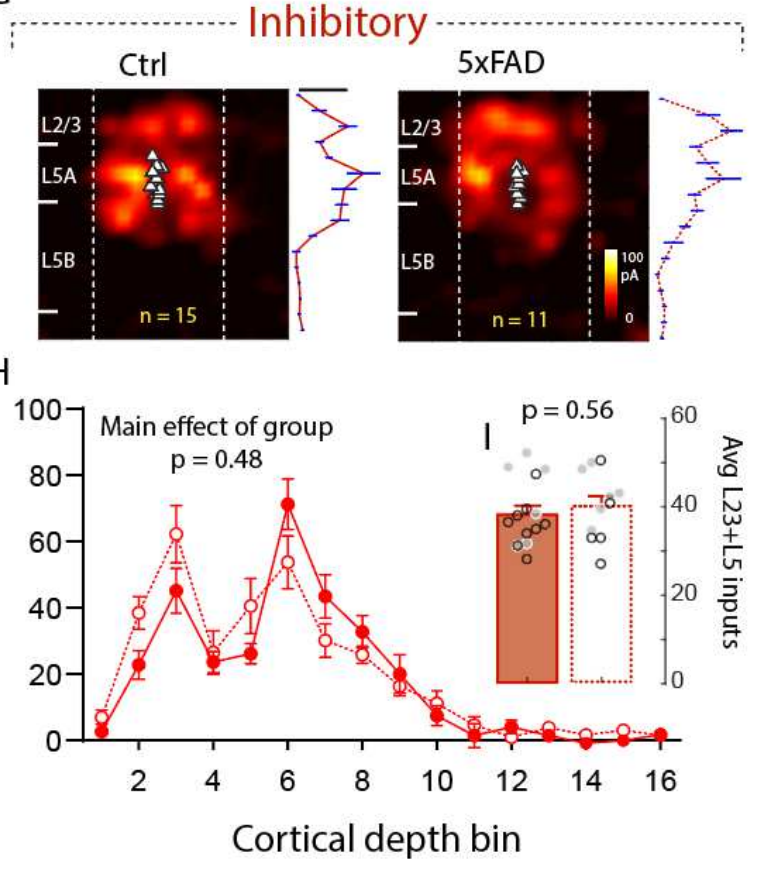